\title{
A Posteriori Validation of Pre-operative Planning in Functional Neurosurgery by Quantification of Brain Pneumocephalus
}

\author{
É. Bardinet ${ }^{1}$, P. Cathier ${ }^{1}$, A. Roche ${ }^{2}$, N. Ayache ${ }^{1}$, and D. Dormont ${ }^{3}$ \\ 1 INRIA, Epidaure Project, Sophia Antipolis, France \\ 2 Robotics Research Laboratory, Dept. of Engineering, University of Oxford, UK \\ 3 Neuroradiology Dept and LENA UPR 640-CNRS, Salpêtrière Hospital, France
}

\begin{abstract}
Functional neurosurgery for Parkinson's disease is based on the stereotactic introduction of electrodes in a small, deeply located, nucleus of the brain. This nucleus is targeted on pre-operative stereotactic MR acquisitions. The procedure is time-consuming and can lead to the development of a pneumocephalus (presence of air in the intracanial cavity) because of CSF leak. This pneumocephalus leads to a brain shift which can yield a significative deformation of the entire brain and thus cause potential errors in the pre-operatively determined position of the stereotactic targets. In this paper, we conduct an a posteriori validation of the pre-operative planning, by quantifying brain pneumocephalus from the registration of pre and immediate post-operative MR acquisitions.
\end{abstract}

\section{Introduction}

MR image guided brain surgery is an actively developping field. In most of the cases, the techniques are based on the use of volumetric pre-operative MR acquisitions. These techniques implicitely assume that a pre-operative acquisition gives a faithful and precise representation of the brain anatomy during the intervention. A major limit of these techniques is the development of brain deformation during the surgical intervention, thus leading to anatomical differences, which can be significative, with the pre-operative MR images. To overcome this limit, there has been recent interest in quatifying brain deformation during neurosurgery 411612.

One interesting example of this type of procedures is functional neurosurgery for Parkinson's disease. This intervention is based on the stereotactic introduction of electrodes in a small, deeply located, nucleus of the brain, called the subthalamic nucleus. This nucleus is targeted on pre-operative stereotactic MR acquisitions. During the intervention, which is performed in the operating room, outside the MR unit, an electrophysiological and clinical study is performed with the electrodes to check the pre-operatively determined target position.

This exploration is time-consuming and can lead to the development of a pneumocephalus (presence of air in the intracanial cavity) because of CSF leak. This pneumocephalus leads to a brain shift which can yield a significative deformation of the entire brain and thus cause potential errors in the pre-operatively determined position of the stereotactic targets. Therefore, computing accurately the deformation induced by the pneumocephalus over the entire brain appears to be a key issue, as it will allow to quantify the deformation occurred around 
the stereotactic targets and lead to a posteriori validation of the pre-operative planning.

We have developped a method to quantify brain deformation from pre and immediate post-operative MR acquisitions, based on rigid and non rigid registrations. Our method is related to the approach developped in [12] for estimating tissue deformation induced by intracranial electrode implantation in patients with epilepsy. Nevertheless, if [12] also used a registration based method, technical solutions provided here are different. The novel non rigid registration algorithm used in this paper is based on a true free form deformation modelisation coupled with a mixed regularization, unlike [12] who used cubic B-splines, thus implicitly limiting the search of the deformations to a specific and reduced transformation space. Therefore, the method presented below allows a finer analysis of the deformation.

\section{Material and Methods}

One patient with bilateral subthalamic lateral implantation was studied. The subthalamic targets were determined one day before the intervention using 3D stereotactic IR-FSPGR MR acquistion. Then, the patient had bilateral implantation of depth electrodes at the level of the subthalamic nuclei. MR control using the same acquistion as pre-operative was performed the day after the implantation. Voxel size of both acquisitions was $0.9375 \times 0.9375 \times 1.3 \mathrm{~mm}$, and image dimension was $256 \times 256 \times 124$. On the control acquisition, presence of air collection was clearly observed at the anterior part of the cranial cavity.

The methodology to quantify brain deformation from these pre and immediate post-operative MR acquisitions consisted in three steps:

1. Robust rigid registration of pre and post-operative MR acquisitions

2. Segmentation of the cortex on the registered images

3. Non-rigid registration of the resulting images

\subsection{Rigid Registration}

We first performed a rigid registration between the pre and post-operative MR acquisitions to correct differences in patient positioning. Notice that in the postoperative acquisition, some parts of the head have been deformed, namely an unknwon but limited part of brain tissue has deformed due to pneumocephalus, and skin and fat presented large deformations, mostly due to swelling. All these deformations could bias the rigid registration.

Therefore a robust variant of the correlation ratio based on the GemanMcClure scale estimator was used as the similarity measure [10]. The assumption underlying the correlation ratio is that there exists an unknown mapping between the MR intensities of the pre and post-operative images. This assumption actually did not hold in the whole image because of the deformed parts of the post-operative image. The use of a robust correlation measure prevented the registration from being biased by such outliers.

\subsection{Cortex Segmentation}

Brain deformation is caused by the pneumocephalus, but changes at the scalp level are not. Thus searching for a non rigid deformation accounting for these 
changes, that is searching for a deformation including discontinuities along the brain-scalp frontier, would be rather complex. Moreover, it could induce errors in the deformation estimate inside the brain. Therefore, before computing the non rigid residual deformation, brain was segmented from the rigidly registered MR images. This was done automatically: thresholding with the CSF grey value, morphological opening, erosion, maximal connected component extraction and dilation.

\section{$2.3 \quad$ Non-rigid Registration}

From the segmented and rigidly registered images, a non rigid registration was performed, using the PASHA algorithm, an iconic feature based algorithm.

Preliminaries. Most of the numerous non rigid registration techniques can be classified according to various criteria [1877. We focus here on one major axis: the motion model expressing the prior knowledge we have on the shape of the transformation, which is used to regularize the registration problem.

It is necessary to impose a motion model to a non rigid registration algorithm, otherwise the motion of a point would be estimated independently of the motion of neighboring points, and we would thus obtain a very discontinuous and unrealistic displacement field. In the field of image registration, we distinguish three kind of motion models: parametric, competitive, and fluid models.

The parametric approach constrains the estimate $T$ of the transformation to belong to some low dimensional transformation space $\mathcal{T}$. Mathematically, if $D(I, J, T)$ is some registration distance between the images $I$ and $J$ registered by $T$, a parametric approach solves the following minimization problem: $\min _{T \in \mathcal{T}} D(I, J, T)$ Among the most popular choices of transformation space, we find rigid and affine groups, and kernels such as thin plate splines or B-splines.

Competitive models rely on the use of a regularization energy $R$ (also called stabilizer) carrying on $T$. Whereas parametric regularization is a binary penalization - no transformation outside the transformation space is allowed, all the transformations inside are equiprobable - the competitive approach penalizes a transformation proportionally to its irregularity measured by the regularization energy $R$ [13]. Competitive algorithms puts in competition (hence the name) $D$ and $R$ by solving the following problem: $\min _{\forall T} D(I, J, T)+R(T)$.

Fluid models also rely on the use of a regularization energy $R$. This time, however, this energy does not carry on the transformation itself, but on its evolution. In a discrete, or iterative, view of the process, the regularization energy carries on the difference between the current and the last estimate of the transformation: at iteration $n>0$, the estimate $T_{n}$ of the transformation is found by minimizing $\min _{\forall T_{n}} D\left(I, J, T_{n}\right)+R\left(T_{n}-T_{n-1}\right)$. The typical example of a fluid approach is the viscoelastic algorithm of Christensen [3].

The PASHA Algorithm. We minimize the following energy: $E(C, T)=$ $S(I, J, C)+\|C-T\|^{2}+R(T)$, where $E$ depends on two variables, $C$ and $T$, that are both vector fields (with one vector per pixel). $C$ is a set of pairings between points: for each point of $I$, it gives a corresponding point in $J$ that attracts this point. $T$ is the estimate of the transformation: it is a smooth vector field (constrained by the regularization energy $R$ ) that is attracted by the 
set of correspondences $C$. In the energy, $S$ is an intensity similarity measure, used to find the correspondences $C$, and $R$ is a regularization energy, used to regularize $T$.

This algorithm is an iconic feature based algorithm, i.e. it is really intermediate between geometric feature based and standard intensity based algorithms. Indeed, on one hand, we search for correspondences $C$ between iconic features in the images, and use a geometric distance to fit the transformation $T$ to $C$. On the other hand, there is no segmentation of the images, as we use an intensity similarity measure to pair features. Note that other iconic feature based algorithms (e.g. the "demons" [14], adaptation of optical flow to registration [5], block matching [8,9]) generally do not minimize a global energy. Consequently, the analysis of the behavior of those algorithms is difficult.

We also propose a mixed competitive/fluid regularization for PASHA. Fluid algorithms are able to recover large displacements, but they often do not preserve image topology for real applications. We therefore use in PASHA a mix of fluid and competitive models in order to recover larger displacements, while still constraining the transformation, and thus avoiding dramatic topology changes. The energy minimized by PASHA at iteration $n$ now becomes:

$$
S\left(I, J, C_{n}\right)+\sigma\left\|C_{n}-T_{n}\right\|^{2}+\sigma \lambda\left[\omega R\left(T_{n}-T_{n-1}\right)+(1-\omega) R\left(T_{n}\right)\right]
$$

where $\omega \in[0,1]$ fixes the relative importance of competitive and fluid regularization ( $\omega=0$ being a pure competitive regularization and $\omega=1$ a pure fluid regularization). The $\lambda$ parameter influences the smoothness of the deformation, and $\sigma$ is related to the noise level in the images.

One could minimize the energy $E$ with respect to $C$ and $T$ simultaneously. However, when the regularization energy $R$ is quadratic, the alternate minimization w.r.t. $C$ and $T$ is appealing, because both partial minimizations are very fast: the minimization w.r.t. $C$ can be done for each pixel separately most of the time, and the second step is easily solved by linear convolution. PASHA is designed on that principle. It minimizes the energy (1) alternatively w.r.t. $C$ and $T$. We start from $T_{0}=\operatorname{Id}$ at iteration 0 ; then, at iteration $n$, the alternated minimization scheme gives the following steps:

- Find $C_{n}$ by minimizing $S\left(I, J, C_{n}\right)+\sigma\left\|C_{n}-T_{n-1}\right\|^{2}$. This is done in PASHA using gradient descent.

- Find $T_{n}$ by minimizing $\left\|C_{n}-T_{n}\right\|^{2}+\lambda\left[\omega R\left(T_{n}-T_{n-1}\right)+(1-\omega) R\left(T_{n}\right)\right]$. This minimization step has a closed-form solution, using convolution.

Let us illustrate the advantage of this mixed regularization with an example: in Fig. 1, we have two noisy images presenting some large motion. Both the recovered deformation field and the intensity differences between the deformed and the original images show that: 1) fluid regularization is able to register the images, but being sensitive to noise, the recovered deformation field is aberrant; 2) competitive regularization is much more robust to noise, but it is more difficult to recover large deformations, as can be seen on the difference image; 3) mixed regularization combines the advantages of both approaches, i.e. good image match while having a regular deformation field.

Finally, the whole minimization process is embeded into a pyramidal framework. This classical technique has two advantages. First, the registration algo- 


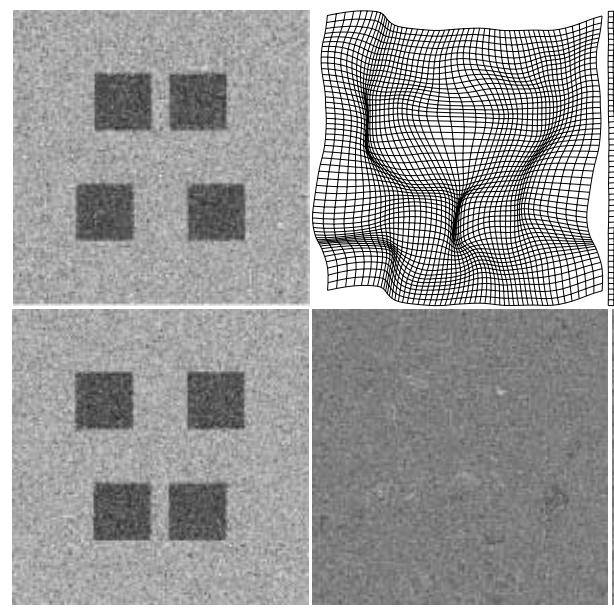

Fluid reg.
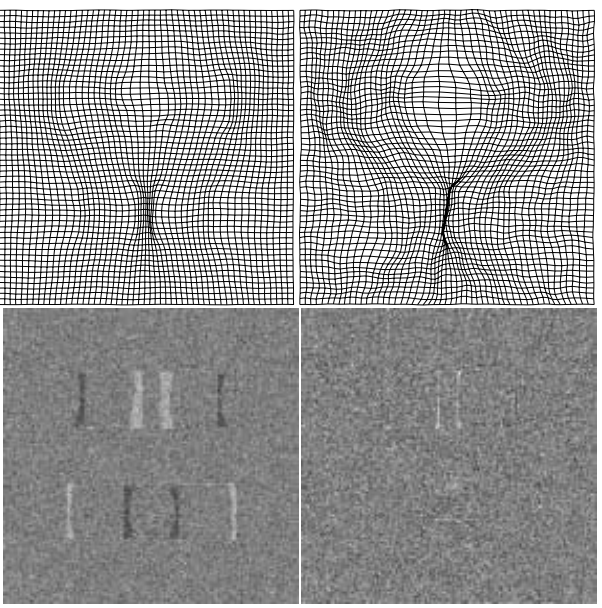

Competitive reg.

Mixed reg.

Fig. 1. Left most column: two noisy images to be registered. Remaining right columns: registration results using PASHA and different regularization techniques. Upper row: Recovered deformation field. Lower row: Intensity differences between the deformed and the original images.

rithm is much less sensitive to initial alignment and can go beyond local minima. Second, the cost of the pyramidal approach is relatively small, since the extra processed images are much smaller than the original one. The similarity measure used in PASHA relies on the computation of local statistics (namely the Gaussian-weighted local correlation coefficient). Local measures assume that the link between $I$ and $J$ is valid only locally. For local statistics, there exists a fast computation method based on convolution that makes it applicable for non rigid registration. For more details, we refer the reader to [2].

\section{Results and Discussion}

Robust rigid registration between the pre and post-operative MR acquisitions was performed first. Fig. 2 shows three independant slices (sagittal, coronal and axial) through both volumes after registration.

As can be seen with the cursor superimposed on the images, correspondences at the bone level are correct, as required because the bone is the only structure which didn't suffer deformation during surgery. Notice that these three slices are not related, as they were independantly selected to illustrate the quality of bone correspondence. Therefore, the cursor location is also independant in each slice. Starting from this rigid registration, the non rigid registration will only search for residual deformations due to pneumocephalus.

To avoid discontinuities along the brain-scalp frontier, brain was extracted from both volumes. Results of this segmentation can be seen in Figs. 3 and 4 .

Then, non rigid registration was performed ( 8 minutes on a $450 \mathrm{Mhz}$ Pentium III), with the PASHA algorithm, including the mixed fluid/competitive regularization, with $\omega=0.6$, which enabled a total recovery of motion by a smooth 

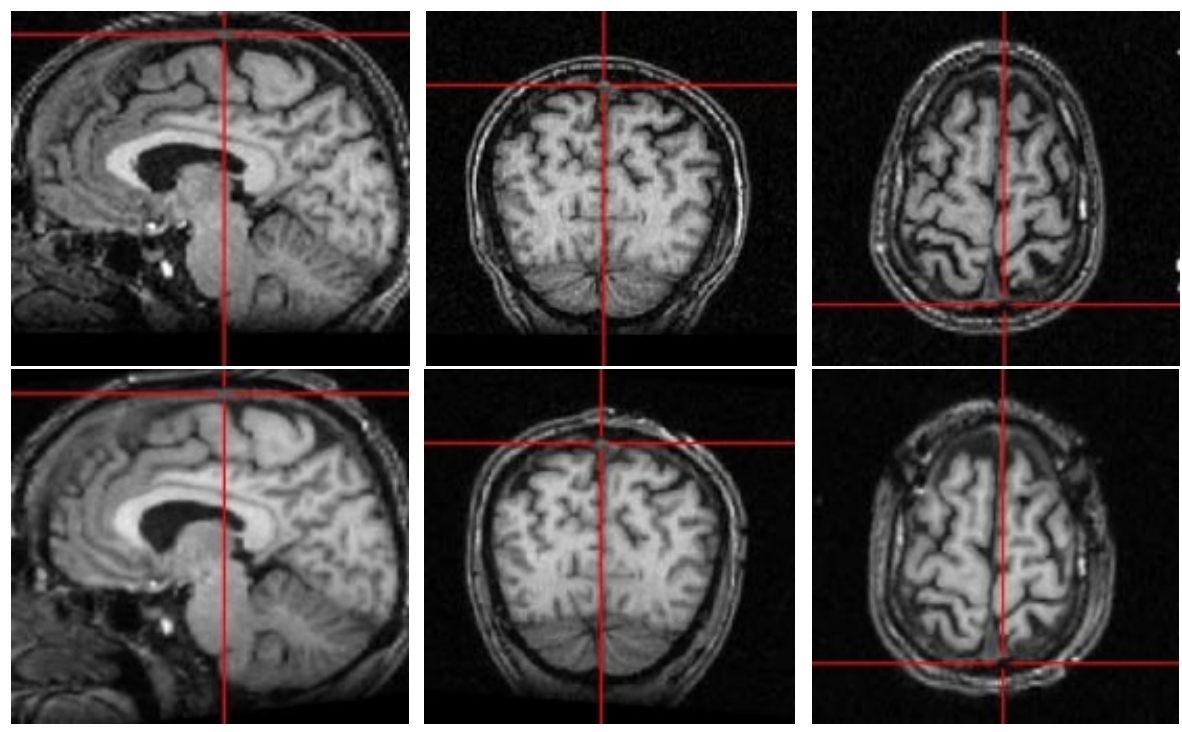

Fig. 2. Robust rigid registration of pre-operative (top row) and post-operative (bottom row) MR images of the same patient. Cursor location illustrates the accurate correspondences found at the bone level. From left to right: independant sagittal, coronal and axial views. Note that the three slices are not related. Therefore, the cursor location is also independant in each slice.

transformation. Fig. 3 shows slices of the post-operative and pre-operative (after rigid and non rigid registration) volumes, with post-operative contours superimposed in order to visually assess the quality of the registration. Notice in particular the consistent matching, both geometrical and textural, obtained around the pre-frontal lobe, where deformation caused by the pneumocephalus was largest. Once the deformation was computed (the maximal value of the deformation norm was $5.79 \mathrm{~mm}$ ), it was possible to examine its spatial distribution, and especially to look at regions of brain that suffered deformations higher than a given value. Fig. 4 shows, on the first row, axial slices through the deformation field itself, the displacement of each voxel being represented by an arrow. Then, on the next two rows, isolines of the deformation norm are superimposed on the same axial slices of the pre-operative volume (after rigid registration and brain extraction) and of the post-operative volume (after brain extraction). The isovalues were set to 3,2 and $1 \mathrm{~mm}$ ( $3 \mathrm{~mm}$ corresponding to the smallest region). As expected, the deformation maximal values coincided with the prefrontal lobe where the pneumocephalus was observed. Then, as can be seen, the deformation smoothly decreased, reaching values under $1 \mathrm{~mm}$ at the basal ganglia level. Also, deformation around the targets (identified by the dark holes located between the putamen and the thalamus on the post-operative images) was significantly lower than $1 \mathrm{~mm}$ (around $0.7 \mathrm{~mm}$ for the anterior part of the targets, and around 0.4 $\mathrm{mm}$ for the posterior part). Note that the localised deformation area (greater than $1 \mathrm{~mm}$ ) at the level of the target on slice 134 was due to the electrode MR signal. 

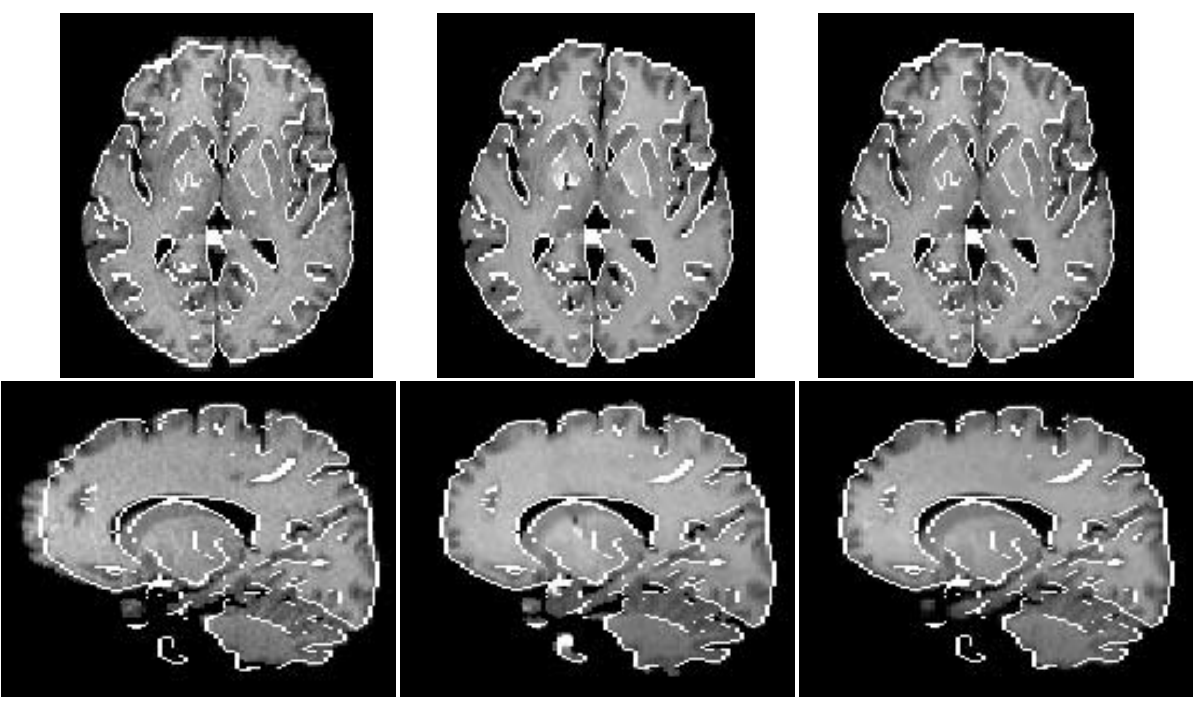

Fig. 3. Non rigid registration of pre-operative and post-operative MR images of the same patient. First row: axial slices; second row: sagittal slices. Left column: preoperative volume after rigid registration; middle column: post-operative volume; right column: pre-operative volume after non rigid registration. Contours of the postoperative volume are superimposed on all the images.

\section{Conclusion}

We addressed the problem of validating a posteriori pre-operative planning in functional neurosurgery. Quantifying per-operative brain deformation around functional targets was done by registration of pre and immediate post-operative MR acquisitions. The non rigid registration algorithm used in this paper was based on a true free form deformation modelisation coupled with a mixed regularization, yielding a faithful deformation field. Analysis of the spatial distribution of brain deformation was then performed. On the patient under study, results confirmed surgeon intuition, i.e. pneumocephalus doesn't affect target pre-operative localisation. Indeed, the deformation was significantly inferior than $1 \mathrm{~mm}$ around the functional targets, knowing that the voxel size of the MR images was around $1 \mathrm{~mm}^{3}$. To confirm the result obtained on this patient, a clinical study will now be conducted on a large series of Parkinsonian patients.

\section{References}

1. L. G. Brown. A Survey of Image Registration Techniques. ACM Computing Surveys, 24(4):325 $-276,121992$.

2. P. Cachier and X. Pennec. 3D Non-Rigid Registration by Gradient Descent on a GaussianWindowed Similarity Measure using Convolutions. In Proc. of MMBIA'00, LNCS 1935, pp. 182 - 189, June 2000.

3. G. E. Christensen, R. D. Rabitt, and M. I. Miller. Deformable Templates Using Large Deformation Kinematics. IEEE TIP, 5(10):1435-1447, October 1996.

4. N. Hata, A. Nabavi, S. Warfield, W. Wells, R. Kikinis, and F.A. Jolesz. A volumetric optical flow method for measurement of brain deformation from intraoperative magnetic resonance images. In Proc. of MICCAI'99, LNCS 1679, September 1999. 


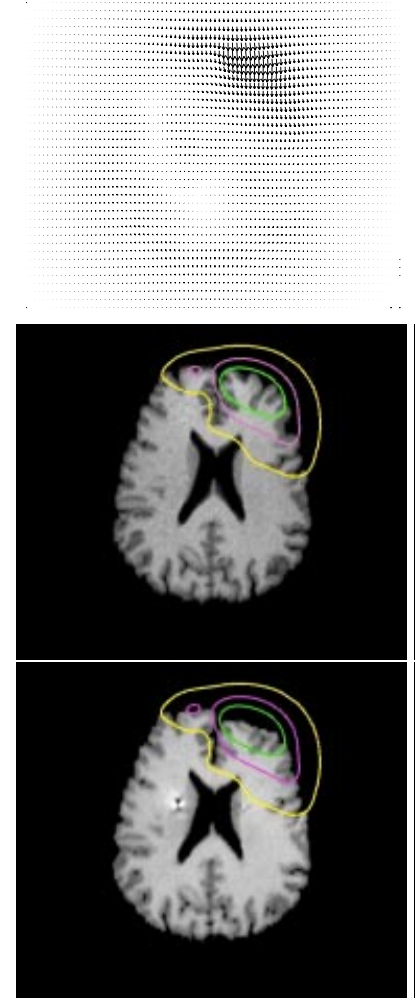

Slice 125

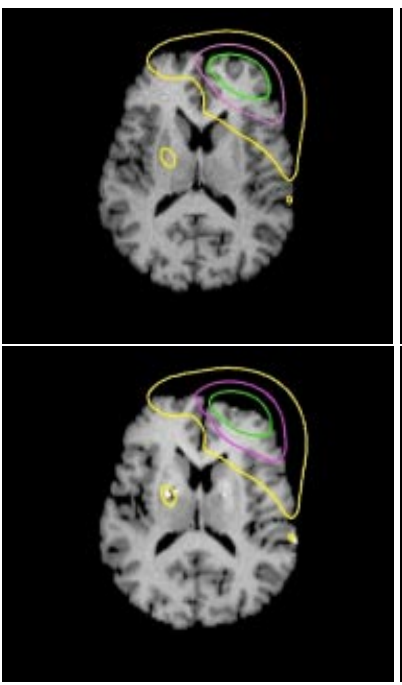

Slice 134

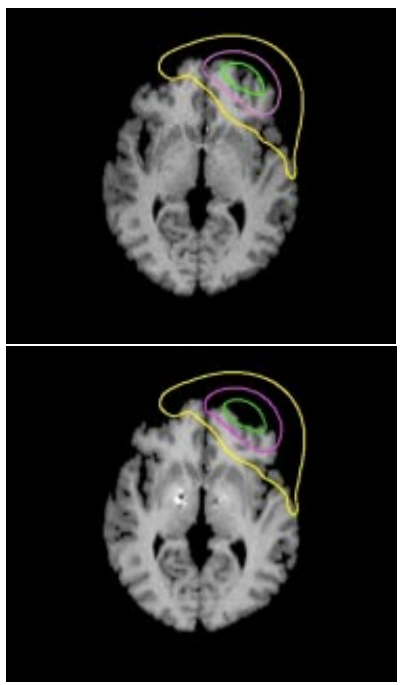

Slice 145

Fig. 4. Non rigid registration of pre and post-operative MR images of the same patient, starting from the rigidly registered images, after brain extraction. Three (columns 1, 2 and 3) axial slices. First row: deformation field; second row: pre-operative image; third row: post-operative image. On the last two rows, isolines of the deformation norm (3, 2, $1 \mathrm{~mm})$ are superimposed on the images.

5. P. Hellier, C. Barillot, E. Mmin, and P. Prez. Medical Image Registration with Robust Multigrid Techniques. In Proc. of MICCAI'99, LNCS 1679, pp. 680 - 687, September 1999.

6. D.L.G. Hill, C.R. Maurer, A.J. Martin, S. Sabanathan, W.A. Hall, D.J. Hawkes, D. Rueckert, and C.L. Truwit. Assessment of Intraoperative Brain Deformation Using Interventional MR Imaging. In Proc. of MICCAI'99, LNCS 1679, pp. 910 - 919, September 1999.

7. H. Lester and S. R. Arridge. A Survey of Hierarchical Non-Linear Medical Image Registration. Pattern Recognition, 32:129 - 149, 1999.

8. J. B. A. Maintz and M. A. Viergever. A Survey of Medical Image Registration. Medical Image Analysis, 2(1):1-36, 1998.

9. S. Ourselin, A. Roche, G. Subsol, X. Pennec, and N. Ayache. Reconstructing a 3D Structure from Serial Histological Sections. IVC, 19(1-2):25-31, January 2001.

10. A. Roche, G. Malandain, and N. Ayache. Unifying Maximum Likelihood Approaches in Medical Image Registration. Int.J.of Imaging Systems and Technology, 11:71-80, 2000.

11. O.M. Skrinjar and J.S. Duncan. Real Time 3D Brain Shift Compensation. In Proc. of IPMI'99, LNCS 1613, pp. 42 - 55, June 1999.

12. C. Studholme, E. Novotny, I.G. Zubal, and J.S. Duncan. Estimating Tissue Deformation between Functional Images Induced by Intracranial Electrode Implantation Using Anatomical MRI. NeuroImage, 13:561 - 576, 2001.

13. R. Szeliski. Bayesian Modeling of Uncertainty in Low-Level Vision. Int. J.of Comp. Vision, 5(3):271 - 301, December 1990.

14. J.-P. Thirion. Image matching as a diffusion process: an analogy with Maxwell's demons. Medical Image Analysis, 2(3):243-260, 1998. 